Research Article

\title{
Theoretical Study on Synchronization of the Counter-Rotating Exciter in the Compound Vibrating Field
}

\author{
Yisen Sun (iD, Ming Su (D), Xu Huang, Rongchen Zhao, Rong Li, and Zhiping Xie \\ School of Mechanical and Electrical Engineering, Guizhou Normal University, 550025 Guiyang, China \\ Correspondence should be addressed to Ming Su; suming629@163.com
}

Received 14 March 2021; Revised 10 June 2021; Accepted 28 June 2021; Published 12 July 2021

Academic Editor: Francesco Bucchi

Copyright (c) 2021 Yisen Sun et al. This is an open access article distributed under the Creative Commons Attribution License, which permits unrestricted use, distribution, and reproduction in any medium, provided the original work is properly cited.

\begin{abstract}
Aiming at the impact of the complex vibration environment generated by the integrated vibration equipment on the vibration testing equipment, this paper proposes a new method to solve the vibratory synchronization problem in the compound vibration environment. A new concept of the compound vibrating field is proposed, and a new simple vibrating system with a single counter-rotating exciter in a compound vibrating field is established. The motion differential equation of the system is established by the integral mean method with small parameters, and then the periodic coefficient differential equation is obtained through linearization. Based on the relevant theory of the second-order differential equation with periodic coefficient, the synchronization criterion and stability criterion of the vibrating system are derived. According to the theoretical criteria, the coupling characteristics of the exciter and the vibrating field are numerically simulated and analyzed, which supports the theoretical results. The proposed compound vibrating field provides a new way for studying vibratory synchronization.
\end{abstract}

\section{Introduction}

Vibration is a common phenomenon in nature, ranging from celestial vibration to heartbeat and breathing. As a new research direction in the field of mechanical vibration, vibration utilization engineering has developed rapidly and has applications in various engineering fields. For example, in medical equipment engineering, ultrasound is used to detect illness; in marine engineering, wave vibration is used to generate electricity; in industrial production, vibration is used for transportation, crushing, and diagnosis [1]. The phenomenon of vibratory synchronization is a ubiquitous and interesting phenomenon in vibration utilization engineering, which has been studied.

The phenomenon of vibratory synchronization was first discovered by the Dutch physicist Huygens through the "pendulum clock experiment" in 1665 [2]. Since then, scientists in various fields have successively discovered synchronization phenomena in the direction of their research. For example, the Dutch physicist van der Pol discovered synchronization in nonlinear circuits and called this phenomenon "frequency capture" [3]. The British physicist
Rayleigh discovered that two organ pipes that are close to each other can simultaneously emit sounds, which provides a basis for perfecting the Rayleigh principle in the future [4].

Until the mid-to-late 20th century, the former Soviet scholar Professor Blekhman applied the method of direct separation of motions to explain the relationship between the relative fast and slow motions of the two eccentric rotors, constructed a dual-exciters vibrating system model, and used the Poincare-Lyapunov small parameter method to verify the stability of the motion system. He proposed the self-synchronization theory of double exciters $[5,6]$. Professor Wen et al. applied the average torque difference method to study the synchronization and stability of the system with the inertia torque difference and phase difference parameters. In 1983, he published several research results of his team on vibration synchronization theory for the first time. He proposed the concept of vibratory synchronization transmission [7-9]. Based on the average torque difference method, Professor Zhao et al. proposed the average method of modified small parameters. He used the disturbance parameter of average angular velocity and average phase difference to convert the synchronization 
problem of the two rotors into a problem on the existence and stability of zero solution for the differential equation. He obtained the conditions for system synchronization and stability and explained the self-synchronization phenomenon of the two rotors [10-12]. In 1997, Professor Blekhman defined synchronization. Synchronization is the correlated or corresponding in-time behavior of two or more processes. And he also defined synchronization in the fields of kinematics and dynamics [13]. Professor Balthazar et al. studied the synchronization of nonideal and nonlinear vibrating system of two exciters, multiple exciters, and other oscillators on the flexible portal frame structure [14-16]. With the perfection of the vibratory synchronization theory system, the vibrating system has expanded from the original dualexciter model to the same-rotation and opposite-rotation models, four-exciter and multiexciter models, dual-mass and multimass models, resonance and superresonance models, and many other systems. Based on the theoretical research methods of predecessors, Zhang et al. [17-19], Kong et al. [20, 21], Gu et al. [22], Liu et al. [23], and others have studied these models and achieved remarkable achievements in engineering applications. However, most scholars have studied vibratory synchronization between dual-exciter and multiexciter models. Due to the complexity of the dual-exciter or multiexciter system, it is difficult to construct the mathematical model of the nonlinear vibrating system, which makes the derivation of the synchronization criterion and the stability criterion have certain limitations $[24,25]$.

Inspired by the abovementioned problems, Professor $\mathrm{Su}$ et al. proposed an integral mean method with small parameters and periodic coefficient (IMM-SPPC) for the vibratory synchronization of a single corotating exciter affected by a single vibration environment and obtained relevant theoretical results [26]. With the large-scale development of equipment, more and more equipment has been integrated and platformization. A lot of equipment will be integrated in a platform, such as the integration of feeders, crushers, screening machines, and other equipment in the building-station crushing workstation [27]. Such the integrated equipment is a huge compound vibrating platform. In this kind of vibration environment, when the angular velocity (angular frequency) of the exciter of the vibration testing equipment is close to the angular frequency of the vibration environment, the angular velocity of the exciter and the angular frequency of the vibration environment will be synchronized. When the angular frequency of the vibration environment changes, it may even cause fluctuations in the angular velocity of the exciter; that is, the exciter will be synchronized with the vibration environment, and the other will not be synchronized. And the exciter has almost no effect on the vibration environment. Due to the complication of the abovementioned working conditions, the problem of the vibratory synchronization of the counterrotating exciter in the compound vibrating field is proposed. Therefore, this paper transforms this situation into the following model and proposes a vibrating system with a single counter-rotating exciter in a compound vibrating field. This research is based on the premise that the influence of the exciter on the vibrating field is negligible. This system is composed of an exciter and a compound vibrating field. The exciter consists of a motor and an eccentric rotor which is driven by the motor to rotate clockwise. The compound vibrating field is composed of vibrating fields that change periodically in the $x$-direction and $y$-direction. The eccentric rotor that rotates clockwise is opposite to the vibration frequency of the vibrating field, so the exciter is a single counter-rotating exciter. The schematic diagram of the vibrating system is shown in Figure 1.

The main contributions and novelties of this paper are summarized as follows:

(1) Based on the complex vibration environment generated by the integrated vibration equipment, the concept of compound vibrating field is proposed, which expands the vibration environment in a single direction and provides a new way for the study of vibration environment.

(2) Through the IMM-SPPC method, the system differential equation is derived to obtain the synchronization criterion and the stability criterion of the new system model, and the two criteria are combined to obtain the range of the phase sum when the vibration system is synchronized and stable, which provides a theoretical reference for the parameter design of vibration testing equipment.

This paper conducts theoretical research and simulation analysis on the vibrating system of the counter-rotating eccentric rotor in the compound vibration field. Section 2 introduces the vibrating system model with a single counterrotating exciter in a compound vibrating field. Section 3 derives the synchronization criterion through the obtained integral mean differential equation with small parameters and derives the stability criterion through the relevant theory of the second-order differential equation with periodic coefficient. In Section 4, based on the derivation of the abovementioned theoretical criteria, the computer numerical simulation of the single exciter vibrating system model is carried out. Finally, some important conclusions are provided.

\section{Modeling of the Vibrating System}

Figure 2 shows the ideal model of the vibrating system. The system is composed of an exciter and a compound vibrating field. Assuming that the rotor with eccentric mass $m$, eccentric radius $r$, and moment of inertia $J$ rotates clockwise around point $O$. The point $O$ satisfies the laws of vibration motion with amplitude $A_{x}$ and $A_{y}$; that is, the vibrating field strength in the $x$-direction and $y$-direction is, respectively, $V_{x}=-\omega^{2} A_{x} \cos \omega t$ and $V_{y}=-\omega^{2} A_{y} \sin \omega t$. And the vibrating field is constant and not affected by the reaction of the motor torque. During the movement, the compound vibrating field causes the exciter to vibrate, so that the eccentric rotor is subjected to vibrating torque.

Due to the vibrating field strength defined in this paper, the exciter vibrates in the counter-rotating exciter at point 


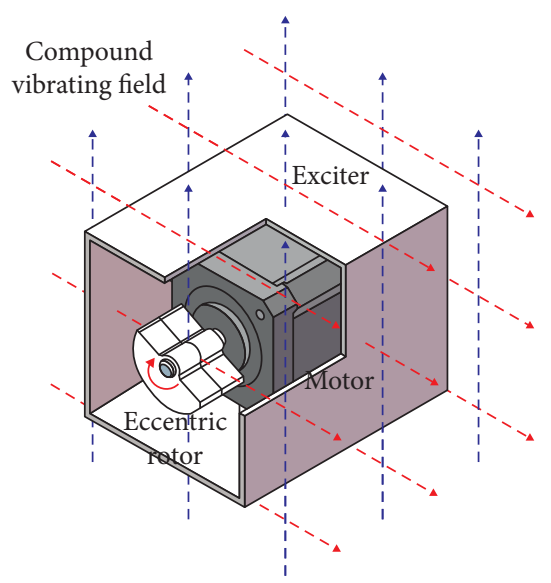

FIGURE 1: Vibrating system of counter-rotating eccentric rotor in compound vibrating field.

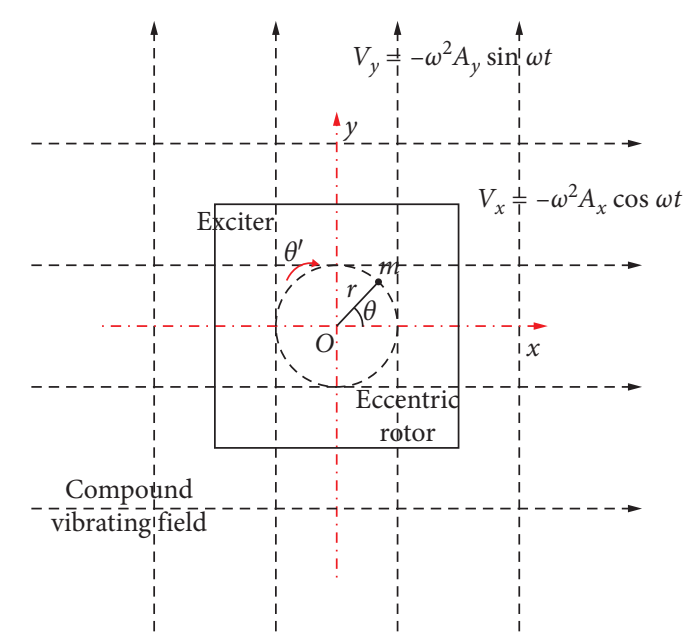

FIGURe 2: Dynamic model of vibrating system with counter-rotating eccentric rotor in compound vibrating field.

$O$, and the equations of motion in the $x$-direction and $y$-direction are, respectively,

$$
\begin{aligned}
& \left\{\begin{aligned}
x & =A_{x} \cos \varphi, \\
x^{\prime} & =-\omega A_{x} \sin \varphi, \quad(\varphi=\omega t), \\
x^{\prime \prime} & =-\omega^{2} A_{x} \cos \varphi,
\end{aligned}\right. \\
& \left\{\begin{aligned}
y & =A_{y} \sin \varphi, \\
y^{\prime} & =\omega A_{y} \cos \varphi, \quad(\varphi=\omega t), \\
y^{\prime \prime} & =-\omega^{2} A_{y} \sin \varphi,
\end{aligned}\right.
\end{aligned}
$$

where $\varphi$ is the angular displacement of vibration, $\omega$ is the rotational angular frequency of vibration, $x, x^{\prime}$, and $x^{\prime \prime}$ are the displacement, velocity, and acceleration of vibration in the $x$-direction, $y, y^{\prime}$, and $y^{\prime \prime}$ are the displacement, velocity, and acceleration of vibration in the $y$-direction, and $A_{x}$ and $A_{y}$ are the amplitudes in the $x$-direction and $y$-direction.
2.1. Dynamic Equations of the Vibrating System. By analyzing the force of the vibrating system, the torque balance equation of the eccentric rotor in compound vibrating field can be obtained as follows:

$$
T_{J}+T_{f}+T_{d}=T_{e}+T_{z} .
$$

So, the dynamic equation of the eccentric rotor is obtained as follows:

$$
\begin{aligned}
T_{J}+T_{f}+T_{d}= & T_{e}-\left(\frac{m r \omega^{2} A_{x}}{2}[\sin (\theta+\varphi)+\sin (\theta-\varphi)]\right) \\
& +\left(\frac{m r \omega^{2} A_{y}}{2}[\sin (\theta+\varphi)-\sin (\theta-\varphi)]\right),
\end{aligned}
$$

where $T_{J}=J \theta^{\prime \prime}$ is the moment of inertia of the eccentric rotor; $T_{f}$ and $T_{d}$ are the friction torque and fan load that the exciter needs to overcome; $T_{e}$ is the electromagnetic torque of the exciter motor. Since the eccentric rotor that rotates clockwise is opposite to the vibration frequency of the vibrating field, therefore, $T_{e}$ is always negative in this paper to indicate the clockwise rotation direction of the exciter; $T_{z}$ is the combined vibrating torque of $T_{x}$ and $T_{y}$ generated by vibration in the $x$-direction and $y$-direction; $T_{x}=m r x^{\prime \prime} \sin \theta=-\left(m r \omega^{2} A_{x} \cos \varphi \sin \theta\right)$ is the vibrating torque of the eccentric rotor in the $x$-direction in vibrating field; $T_{y}=-m r y^{\prime \prime} \cos \theta=m r \omega^{2} A_{y} \sin \varphi \cos \theta$ is the vibrating torque of the eccentric rotor in the $y$-direction in vibrating field; $\theta$ is the angular displacement of the eccentric rotor, which is a negative value because it rotates clockwise.

2.2. Differential Equations for the Relative Motion of the Vibrating System. In this system, the rotational motion of the eccentric rotor and the vibration of the vibrating field affect each other, as shown in Figure 3. This paper is based on the vibration of the vibrating field. Therefore, we use the interaction of the two motions in a period of the vibrating field as a unit to study the relative motion between the two motions.

Assumption: the eccentric rotor can be captured by the vibrating field, and some hypothetical conclusions can be obtained as follows:

(1) The angular velocity of the eccentric rotor $\theta$ is captured by the angular frequency of the vibrating field $\omega, \theta_{0}=\omega$.

(2) The sum of the angular displacement of the eccentric rotor $\theta$ and the angular displacement of vibration $\varphi$ is the phase sum $\Delta \alpha, \theta+\varphi=\Delta \alpha$, where $\Delta \alpha$ is a constant that can be converted in the range of $[0,2 \pi]$.

(3) The mean angular velocity of the eccentric rotor $\theta^{\prime}$ and the mean angular velocity of the vibrating field $\omega$ are equal in the integration period $[t, t+2 \pi / \omega]$, and the rotation direction is opposite, $(\omega / 2 \pi) \int_{t}^{t+(2 \pi / \omega)}$ $\left(\theta+\varphi^{\prime}\right) \mathrm{d} t=0$. 


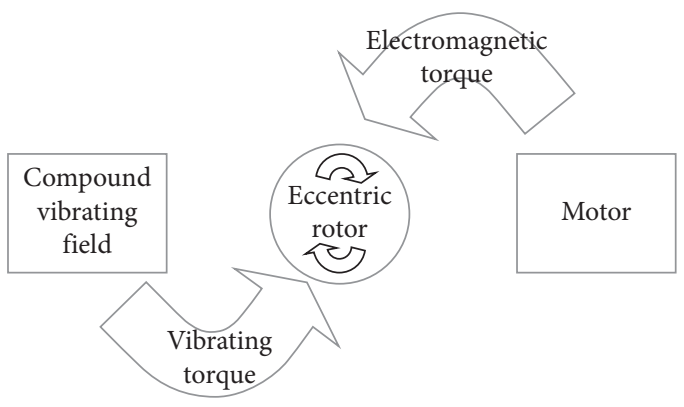

Figure 3: Vibration and rotation coupled system.

Assume that the integral mean variable with small parameters of the phase sum is $\bar{\varepsilon}$, that is, at the equilibrium point, the difference between the integral mean of the sum of the angular displacement $\theta$ of the eccentric rotor and the angular displacement $\varphi$ of the vibration in the vibrating period, and the phase sum $\Delta \alpha$ during synchronization. It can be expressed as follows:

$$
\bar{\varepsilon}(t)=\frac{\omega}{2 \pi} \int_{t}^{t+(2 \pi / \omega)}(\theta+\varphi) \mathrm{d} t-\Delta \alpha,
$$

with

$$
\begin{aligned}
& \bar{\varepsilon}^{\prime}(t)=\frac{\omega}{2 \pi} \int_{t}^{t+(2 \pi / \omega)}\left(\theta^{\prime}+\varphi^{\prime}\right) \mathrm{d} t, \\
& \bar{\varepsilon}^{\prime \prime}(t)=\frac{\omega}{2 \pi} \int_{t}^{t+(2 \pi / \omega)} \theta^{\prime \prime} \mathrm{d} t .
\end{aligned}
$$

When the vibrating system is synchronized and stable, $(\omega / 2 \pi) \int_{t}^{t+(2 \pi / \omega)} \bar{\varepsilon}(t) \mathrm{d} t=0, \bar{\varepsilon}(t) \ll 1$.

At the torque equilibrium point,

$$
\begin{aligned}
& T_{e} \approx T_{e_{0}}-C_{M}\left(\theta^{\prime}+\omega\right), \\
& T_{d} \approx T_{d_{0}}-m f d r \omega\left(\theta^{\prime}+\omega\right), \\
& T_{f} \approx T_{f_{0}}-f\left(\theta^{\prime}+\omega\right),
\end{aligned}
$$

where $T_{e_{0}}$ is the electromagnetic torque and is a negative value when the eccentric rotor is captured by the vibrating field; $C_{M}=\mathrm{d} T_{M} /\left.\mathrm{d} \theta^{\prime}\right|_{\omega}$ is the slope of the mechanical characteristic curve and is a positive value when the eccentric rotor is captured by the vibrating field; $T_{d_{0}}=-\left(m r \omega^{2} f d / 2\right)$ is the load of the fan and is a positive value when the eccentric rotor is captured by the vibrating field; $d$ is the equivalent diameter of the fan load of the exciter; $T_{f_{0}}=-f \omega$ is the friction torque and is a positive value when the eccentric rotor is captured by the vibrating field; $f$ is the coefficient of friction.

In order to better study the relationship between the two motions in the dynamic equation, this paper uses $\bar{\varepsilon}(t)$ as a variable and transforms (4) and integrates it in $[t, t+(2 \pi / \omega)]$, which can be obtained as follows:

$$
\begin{aligned}
\bar{\varepsilon}^{\prime \prime}(t)+\left(\frac{C_{M}-f-m f d r \omega}{J}\right) \bar{\varepsilon}^{\prime}(t)+\left(\frac{T_{f_{0}}+T_{d_{0}}-T_{e_{0}}}{J}\right) \\
=-\frac{m r \omega^{2} A_{x}}{2 J}\left[\frac{\omega}{2 \pi} \int_{t}^{t+(2 \pi / \omega)} \sin (\theta+\varphi) \mathrm{d} t+\frac{\omega}{2 \pi} \int_{t}^{t+(2 \pi / \omega)} \sin (\theta-\varphi) \mathrm{d} t\right] \\
+\frac{m r \omega^{2} A_{y}}{2 J}\left[\frac{\omega}{2 \pi} \int_{t}^{t+(2 \pi / \omega)} \sin (\theta+\varphi) \mathrm{d} t-\frac{\omega}{2 \pi} \int_{t}^{t+(2 \pi / \omega)} \sin (\theta-\varphi) \mathrm{d} t\right]
\end{aligned}
$$

Equation (9) is the differential equation of motion of the vibrating system.

This section describes the mathematical model of the vibrating system of counter-rotating exciter in compound vibrating field. The differential equation of motion of the vibrating system is obtained by the integral mean method with small parameter, which lays a foundation for the study of synchronization and stability of the system.

\section{Synchronization Criterion and Stability Criterion of Vibrating System}

3.1. Linearization of the Equation. Equation (9) has a trigonometric function containing the relationship between the $\theta$ and $\varphi$ variables, which needs to be transformed into a unified variable, so the trigonometric function is linearized and transformed into a differential equation containing $\bar{\varepsilon}(t)$ variable. Since this paper finally studies the model at the equilibrium point of $\theta+\varphi=\Delta \alpha$, the trigonometric function in (9) should be linearized at $\theta+\varphi=\Delta \alpha$.

3.1.1. $(\omega / 2 \pi) \int_{t}^{t+(2 \pi / \omega)} \sin (\theta+\varphi) d t \quad$ of Linearization. Applying Taylor's formula to $\sin (\theta+\varphi)$ at $\theta+\varphi=\Delta \alpha$, ignoring the higher-order terms, it can be obtained as follows:

$$
\sin (\theta+\varphi)=\sin \Delta \alpha+(\theta+\varphi-\Delta \alpha) \cos \Delta \alpha
$$

Integrate equation (10) to get

$$
\begin{aligned}
& \frac{\omega}{2 \pi} \int_{t}^{t+(2 \pi / \omega)} \sin (\theta+\varphi) \mathrm{d} t \\
& \quad=\sin \Delta \alpha+\frac{\omega}{2 \pi} \cos \Delta \alpha \int_{t}^{t+(2 \pi / \omega)}(\theta+\varphi-\Delta \alpha) \mathrm{d} t
\end{aligned}
$$

Noticed (5),

$$
\frac{\omega}{2 \pi} \int_{t}^{t+(2 \pi / \omega)}(\theta+\varphi-\Delta \alpha) \mathrm{d} t=\bar{\varepsilon}(t)
$$

Substituting (12) into (11), it can be obtained as follows:

$$
\frac{\omega}{2 \pi} \int_{t}^{t+(2 \pi / \omega)} \sin (\theta+\varphi) \mathrm{d} t=\sin \Delta \alpha+\bar{\varepsilon}(t) \cos \Delta \alpha .
$$


3.1.2. $(\omega / 2 \pi) \int_{t}^{t+(2 \pi / \omega)} \sin (\theta-\phi) d t \quad$ of Linearization. Applying Taylor's formula to $\sin (\theta-\varphi)$ at $\theta+\varphi=\Delta \alpha$, ignoring the higher-order terms, it can be obtained as follows:

$\sin (\theta-\varphi)=\sin (\Delta \alpha-2 \varphi)+(\theta+\varphi-\Delta \alpha) \cos (\Delta \alpha-2 \varphi)$.

Integrate equation (14) to get

$$
\begin{aligned}
\frac{\omega}{2 \pi} \int_{t}^{t+(2 \pi / \omega)} \sin (\theta-\varphi) \mathrm{d} t= & -\frac{1}{2 \omega}\left\{\left.\frac{\omega}{2 \pi}[(\theta+\varphi-\Delta \alpha) \sin (\Delta \alpha-2 \varphi)]\right|_{t} ^{t+(2 \pi / \omega)}\right. \\
& \left.-\frac{\omega}{2 \pi} \int_{t}^{t+(2 \pi / \omega)} \sin (\Delta \alpha-2 \varphi) d(\theta+\varphi)\right\} .
\end{aligned}
$$

Noticed (6),

$$
\left.\frac{\omega}{2 \pi}[(\theta+\varphi-\Delta \alpha) \sin (\Delta \alpha-2 \varphi)]\right|_{t} ^{t+(2 \pi / \omega)}=\bar{\varepsilon}^{\prime}(t) \sin (\Delta \alpha-2 \varphi) .
$$

Substituting (16) into (15), it can be obtained as follows:

$$
\begin{aligned}
& \frac{\omega}{2 \pi} \int_{t}^{t+(2 \pi / \omega)} \sin (\theta-\varphi) \mathrm{d} t=-\frac{1}{2 \omega} \bar{\varepsilon}^{\prime}(t) \sin (\Delta \alpha-2 \varphi)+\left(\frac{1}{2 \omega}\right)^{2} \\
& \left\{\left.\frac{\omega}{2 \pi}\left[\left(\theta^{\prime}+\omega\right) \cos (\Delta \alpha-2 \varphi)\right]\right|_{t} ^{t+(2 \pi / \omega)}-\frac{\omega}{2 \pi} \int_{t}^{t+2 \pi / \omega} \cos (\Delta \alpha-2 \varphi) d\left(\theta^{\prime}+\varphi^{\prime}\right)\right\} . \\
& \frac{m r\left(A_{x}+A_{y}\right) \cos (2 \varphi-\Delta \alpha)+8 J}{8 J} \bar{\varepsilon}^{\prime \prime}(t) \\
& +\left(\frac{m r \omega\left(A_{x}+A_{y}\right) \sin (2 \varphi-\Delta \alpha)+4\left(C_{M}-f-m f d r \omega\right)}{4 J}\right) \bar{\varepsilon}^{\prime}(t) \\
& +\frac{m r \omega^{2}\left(A_{x}-A_{y}\right) \cos \Delta \alpha}{2 J} \bar{\varepsilon}(t) \\
& +\left(\frac{m r \omega^{2}\left(A_{x}-A_{y}\right)}{2 J} \sin \Delta \alpha-\frac{T_{e_{0}}-T_{f_{0}}-T_{d_{0}}}{J}\right)=0 .
\end{aligned}
$$

$$
\begin{aligned}
\frac{\omega}{2 \pi} \int_{t}^{t+2 \pi / \omega} \sin (\theta-\varphi) \mathrm{d} t= & -\frac{1}{2 \omega} \bar{\varepsilon}^{\prime}(t) \sin (\Delta \alpha-2 \varphi) \\
& +\left(\frac{1}{2 \omega}\right)^{2} \bar{\varepsilon}^{\prime \prime}(t) \cos (\Delta \alpha-2 \varphi)-\left(\frac{1}{2 \omega}\right)^{2} \\
& {\left[\frac{\omega}{2 \pi} \int_{t}^{t+2 \pi / \omega} \cos (\Delta \alpha-2 \varphi) d\left(\theta^{\prime}+\varphi^{\prime}\right)\right] . }
\end{aligned}
$$

We can recursively conclude that $(\omega / 2 \pi) \int_{t}^{t+(2 \pi / \omega)} \sin (\theta-\varphi) \mathrm{d} t$ is an infinite-order function of $\bar{\varepsilon}^{\prime}(t), \bar{\varepsilon}^{\prime \prime}(t) \ldots$ and ignore the higher-order differential terms above $\bar{\varepsilon}$ second-order differential terms, and it can be obtained as follows:

$$
\begin{aligned}
\frac{\omega}{2 \pi} \int_{t}^{t+2 \pi / \omega} \sin (\theta-\varphi) \mathrm{d} t \approx & \frac{1}{2 \omega} \bar{\varepsilon}^{\prime}(t) \sin (2 \varphi-\Delta \alpha) \\
& +\left(\frac{1}{2 \omega}\right)^{2} \bar{\varepsilon}^{\prime \prime}(t) \cos (2 \varphi-\Delta \alpha) .
\end{aligned}
$$

Incorporating (13) and (20) into (9), the second-order differential equation with periodic coefficient of eccentric rotor in vibrating field, it can be obtained as follows:
This part finally obtains differential (21) with periodic coefficient, which makes it better to satisfy the synchronization and stability conditions when studying the synchronization and stability of the system.

3.2. Synchronization Criterion of Vibrating System. When the exciter is synchronized with the compound vibrating field,

$$
\bar{\varepsilon}^{\prime \prime}(t)=\bar{\varepsilon}^{\prime}(t)=\bar{\varepsilon}(t)=0 .
$$

According to the second-order differential equation with periodic coefficient in Section 3.1, (22) is inserted into (21). The static torque balance equation at the equilibrium point can be obtained as follows:

$$
\frac{m r \omega^{2}\left(A_{x}-A_{y}\right)}{2 J} \sin \Delta \alpha-\frac{T_{e_{0}}-T_{f_{0}}-T_{d_{0}}}{J}=0,
$$

with 


$$
\sin \Delta \alpha=\frac{2\left(T_{e_{0}}-T_{f_{0}}-T_{d_{0}}\right)}{m r \omega^{2}\left(A_{x}-A_{y}\right)} \quad\left(A_{x} \neq A_{y}\right) .
$$

The synchronicity criterion that the angular frequency of the counter-rotating eccentric rotor is captured by the frequency of the vibrating field can be obtained as follows:

$$
\left|\frac{2\left(T_{e_{0}}-T_{f_{0}}-T_{d_{0}}\right)}{m r \omega^{2}\left(A_{x}-A_{y}\right)}\right|<1 .
$$

Due to considering the different working conditions produced by the relationship between the angular frequency of the vibrating field $\omega$ and the no-load angular velocity of the exciter $\omega_{0}$.

When the exciter is operating in an electric working state, the angular frequency of the vibrating field $\omega$ is less than the no-load angular velocity of the exciter $\omega_{0}$, and the amplitude of the vibrating field in the $x$-direction $A_{x}$ is greater than the amplitude in the $y$-direction $A_{y}$, with

$$
\left\{\begin{array}{l}
\omega<\omega_{0} \\
A_{x}>A_{y} .
\end{array}\right.
$$

The relevant conclusions can be obtained as follows:

$$
\begin{aligned}
\left(T_{e_{0}}-T_{f_{0}}-T_{d_{0}}\right) & <0, \\
\sin \Delta \alpha & <0, \\
\bar{T}_{z 0} & =-\frac{m r \omega^{2}\left(A_{x}-A_{y}\right)}{2} \sin \Delta \alpha>0,
\end{aligned}
$$

where $T_{\underline{e} 0}, T_{d 0}$, and $T_{f 0}$ are all negative values, the vibration torque $\bar{T}_{z 0}$ is an average load torque during frequency capture, and the synchronization phase sum $\Delta \alpha$ could be in the third or fourth quadrant.

When the exciter is operating in an electric working state, the angular frequency of the vibrating field $\omega$ is less than the no-load angular velocity of the exciter $\omega_{0}$, and the amplitude of the vibrating field in the $x$-direction $A_{x}$ is less than the amplitude in the $y$-direction $A_{y}$, with

$$
\left\{\begin{array}{l}
\omega<\omega_{0} \\
A_{x}<A_{y}
\end{array}\right.
$$

The relevant conclusions can be obtained as follows:

$$
\begin{aligned}
\left(T_{e_{0}}-T_{f_{0}}-T_{d_{0}}\right) & <0, \\
\sin \Delta \alpha & >0, \\
\bar{T}_{z 0} & =-\left(\frac{m r \omega^{2}\left(A_{x}-A_{y}\right)}{2}\right) \sin \Delta \alpha>0 .
\end{aligned}
$$

where the vibration torque $\bar{T}_{z 0}$ is an average load torque during frequency capture, and the synchronization phase sum $\Delta \alpha$ could be in the first or second quadrant.

When the exciter is operating in a feedback state, the angular frequency of the vibrating field $\omega$ is greater than the no-load angular velocity of the exciter $\omega_{0}$, and the amplitude of the vibrating field in the $x$-direction $A_{x}$ is greater than the amplitude in the $y$-direction $A_{y}$,with

$$
\left\{\begin{array}{l}
\omega>\omega_{0}, \\
A_{x}>A_{y} .
\end{array}\right.
$$

The relevant conclusions can be obtained as follows:

$$
\begin{aligned}
\left(T_{e_{0}}-T_{f_{0}}-T_{d_{0}}\right) & >0 \\
\sin \Delta \alpha & >0 \\
\bar{T}_{z 0} & =-\left(\frac{m r \omega^{2}\left(A_{x}-A_{y}\right)}{2}\right) \sin \Delta \alpha<0,
\end{aligned}
$$

where the vibration torque $\bar{T}_{z 0}$ is an average active torque during frequency capture, and the synchronization phase sum $\Delta \alpha$ could be in the first or second quadrant.

When the exciter is operating in a feedback state, the angular frequency of the vibrating field $\omega$ is greater than the no-load angular velocity of the exciter $\omega_{0}$, and the amplitude of the vibrating field in the $x$-direction $A_{x}$ is less than the amplitude in the $y$-direction $A_{y}$, with

$$
\left\{\begin{array}{l}
\omega>\omega_{0}, \\
A_{x}<A_{y} .
\end{array}\right.
$$

The relevant conclusions can be obtained as follows:

$$
\begin{aligned}
\left(T_{e_{0}}-T_{f_{0}}-T_{d_{0}}\right) & >0, \\
\sin \Delta \alpha & <0, \\
\bar{T}_{z 0} & =-\frac{m r \omega^{2}\left(A_{x}-A_{y}\right)}{2} \sin \Delta \alpha<0,
\end{aligned}
$$

where the vibration torque $\bar{T}_{z 0}$ is an average active torque during frequency capture, and the synchronization phase sum $\Delta \alpha$ could be in the third or fourth quadrant.

In this part, considering the vibrating field and the working conditions of the exciter, the synchronization criterion $\left|2\left(T_{M 0}-T_{d 0}-T_{f 0}\right) / m r \omega^{2}\left(A_{x}-A_{y}\right)\right|<1$ can be used to determine the quadrant distribution of the phase sum $\Delta \alpha$ during the system synchronization.

3.3. Stability Criterion of Vibrating System. When the angular frequency of the eccentric rotor is captured by the frequency of the vibrating field, the differential equation 
with periodic coefficient at the equilibrium point can be obtained as follows:

$$
\begin{aligned}
& \bar{\varepsilon}^{\prime \prime}(t)+\left(\frac{8\left(C_{M}-f-m f d r \omega\right)+2 m r \omega\left(A_{x}+A_{y}\right) \sin (2 \varphi-\Delta \alpha)}{8 J+m r\left(A_{x}+A_{y}\right) \cos (2 \varphi-\Delta \alpha)}\right) \bar{\varepsilon}^{\prime}(t) \\
& +\frac{4 m r \omega^{2}\left(A_{x}-A_{y}\right) \cos \Delta \alpha}{8 J+m r\left(A_{x}+A_{y}\right) \cos (2 \varphi-\Delta \alpha)} \bar{\varepsilon}(t)=0 .
\end{aligned}
$$

Equation (34) is a second-order linear differential equation with periodic coefficients with a period of $\pi / \omega$. According to the relevant theory of the second-order differential equation with periodic coefficient $[28,29]$, if (34) is to reach a stable state, the following two conditions must be met:

$$
\left\{\begin{array}{l}
\frac{4 m r \omega^{2}\left(A_{x}-A_{y}\right) \cos \Delta \alpha}{8 J+m r\left(A_{x}+A_{y}\right) \cos (2 \varphi-\Delta \alpha)}>0, \\
\int_{0}^{\pi / \omega} \frac{8\left(C_{M}-f-m f d r \omega\right)+2 m r \omega\left(A_{x}+A_{y}\right) \sin (2 \varphi-\Delta \alpha)}{8 J+m r\left(A_{x}+A_{y}\right) \cos (2 \varphi-\Delta \alpha)} \mathrm{d} t>0 .
\end{array}\right.
$$

Under normal circumstances, the condition is stratified, $8 J>m r\left(A_{x}+A_{y}\right)$.

From the first condition, it can be derived as follows:

$$
\left\{\begin{array}{l}
\text { When } A_{x}>A_{y}, \cos \Delta \alpha>0, \\
\text { When } A_{x}<A_{y}, \cos \Delta \alpha<0 .
\end{array}\right.
$$

From the second condition, it can be derived as follows:

$$
\begin{aligned}
\int_{0}^{\pi / \omega} & \frac{8\left(C_{M}-f-m f d r \omega\right)+2 m r \omega\left(A_{x}+A_{y}\right) \sin (2 \varphi-\Delta \alpha)}{8 J+m r\left(A_{x}+A_{y}\right) \cos (2 \varphi-\Delta \alpha)} \mathrm{d} t \\
= & \frac{8 \pi\left(C_{M}-f-m f d r \omega\right)}{\omega \sqrt{64 J^{2}-m^{2} r^{2}\left(A_{x}+A_{y}\right)^{2}}}>0 .
\end{aligned}
$$

In summary, the stability criterion can be obtained as follows:

When $A_{x}>A_{y}, \cos \Delta \alpha>0$, synchronization phase sum $\Delta \alpha$ could be in the first or fourth quadrant;

When $A_{x}<A_{y}, \cos \Delta \alpha<0$, synchronization phase sum $\Delta \alpha$ could be in the second or third quadrant.

In this part, considering the vibrating field and the working conditions of the exciter, the stability criterion can be used to determine the quadrant distribution of the phase sum $\Delta \alpha$ during the system stability.

\section{Simulation Analysis of Vibrating System}

This section sets up some quantitative values for simulation analysis to illustrate the effectiveness and feasibility of the above theoretical results.
4.1. Setting Parameters. In this part, by referring to the actual exciter model VB-326-W $(380 \mathrm{~V}, 50 \mathrm{~Hz}, 6$-pole, rated current $0.82 \mathrm{~A}$, rated speed $980 \mathrm{r} / \mathrm{min}$, rated power $0.2 \mathrm{~kW}$, excitation force $0-3 \mathrm{kN}$ ), the structural parameters of the vibrating system are designed, as shown in Table 1.

4.2. Simulation and Analysis. Based on the above design parameters, a simulation model is established in MATLAB/ Simulink.

Simulation 1. The parameters of the system are as follows: $\omega=32.67 \pi \mathrm{rad} / \mathrm{s}, A_{x}=0.005$, and $A_{y}=0.001$.

Verify the synchronization criterion according to the following parameters, when $\omega=32.67 \pi \mathrm{rad} / \mathrm{s}$, we get slip rate $s=0.02, \quad T_{e_{0}}=-2.060, \quad T_{f_{0}}=-f \omega \approx-0.205$, $T_{d_{0}}=-m r \omega^{2} f d / 2 \approx-0.211$, and $m r \omega^{2}\left(A_{x}-A_{y}\right)=8.427$, and we get

$$
\left|\frac{2\left(T_{e_{0}}-T_{f_{0}}-T_{d_{0}}\right)}{m r \omega^{2}\left(A_{x}-A_{y}\right)}\right| \approx 0.390<1 .
$$

The above values satisfy the criterion of synchronization, and the phase sum stable operating point is in the fourth quadrant. The simulation results are shown in Figure 4.

The exciter is in an electric working state, and the synchronization phase sum $\Delta \alpha \approx 62.31 \mathrm{rad}$ (approximately $330.1^{\circ}$ in the range of $360^{\circ}$ ) is in the fourth quadrant.

Simulation 2. The parameters of the system are as follows: $\omega=34 \pi \mathrm{rad} / \mathrm{s}, A_{x}=0.005$, and $A_{y}=0.001$.

Verify the synchronization criterion according to the following parameters, when $\omega=34 \pi \mathrm{rad} / \mathrm{s}$, we get slip rate $s=-0.02, T_{e_{0}}=2.060, T_{f_{0}}=-f \omega \approx-0.214, T_{d_{0}}=$ $-m r \omega^{2} f d / 2 \approx-0.228$, and $m r \omega^{2}\left(A_{x}-A_{y}\right)=9.127$, and we get

$$
\left|\frac{2\left(T_{e_{0}}-T_{f_{0}}-T_{d_{0}}\right)}{m r \omega^{2}\left(A_{x}-A_{y}\right)}\right| \approx 0.548<1 .
$$

The above values satisfy the criterion of synchronization, and the phase sum stable operating point is in the first quadrant. The simulation results are shown in Figure 5.

The exciter is in a feedback state, and the synchronization phase sum $\Delta \alpha \approx 69.4 \mathrm{rad}$ (approximately $16^{\circ}$ in the range of $360^{\circ}$ ) is in the first quadrant.

Simulation 3. The parameters of the system are as follows: $\omega=32.67 \pi \mathrm{rad} / \mathrm{s}, A_{x}=0.001$, and $A_{y}=0.005$.

Verify the synchronization criterion according to the following parameters, when $\omega=32.67 \pi \mathrm{rad} / \mathrm{s}$, we get slip rate $s=0.02, \quad T_{e_{0}}=-2.060, \quad T_{f_{0}}=-f \omega \approx-0.205$, $T_{d_{0}}=-m r \omega^{2} f d / 2 \approx-0.211, \quad$ and $m r \omega^{2}\left(A_{x}-A_{y}\right)=$ -8.427 , and we get 
TABLE 1: Parameters of the vibrating system.

\begin{tabular}{lc}
\hline Parameters & Value \\
\hline Maximum torque of exciter motor $T_{e_{M}}(\mathrm{~N} \cdot \mathrm{m})$ & 3.90 \\
Maximum slip of exciter motor $S_{M}$ & 0.07 \\
No-load angular velocity of exciter $\omega_{0}(\mathrm{r} / \mathrm{min})$ & 1000 \\
Mass of eccentric rotor $m(\mathrm{~kg})$ & 4 \\
Rotation radius of eccentric rotor $r(\mathrm{~m})$ & 0.05 \\
Equivalent diameter of exciter fan load $d(\mathrm{~m})$ & 0.1 \\
Coefficient of friction $f$ & 0.002 \\
Moment of inertia of eccentric rotor $J\left(\mathrm{~kg} \cdot \mathrm{m}^{2}\right)$ & 0.01 \\
Simulation time $T(\mathrm{~s})$ & 100
\end{tabular}

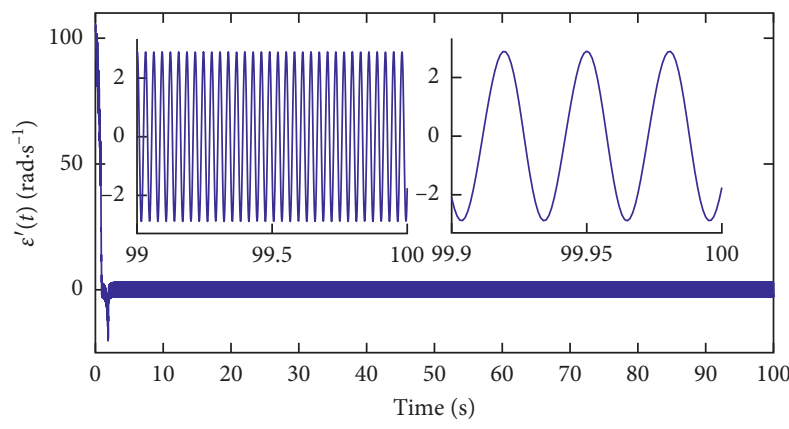

(a)

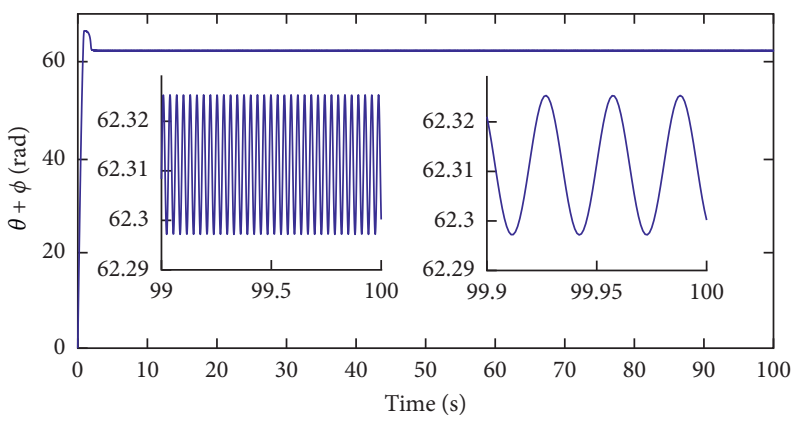

(b)

Figure 4: Simulation curve $\left(\omega=32.67 \pi, A_{x}=0.005\right.$, and $\left.A_{y}=0.001\right)$. (a) $\varepsilon^{\prime}(t)$ graph. (b) $\theta+\varphi(t)$ graph.

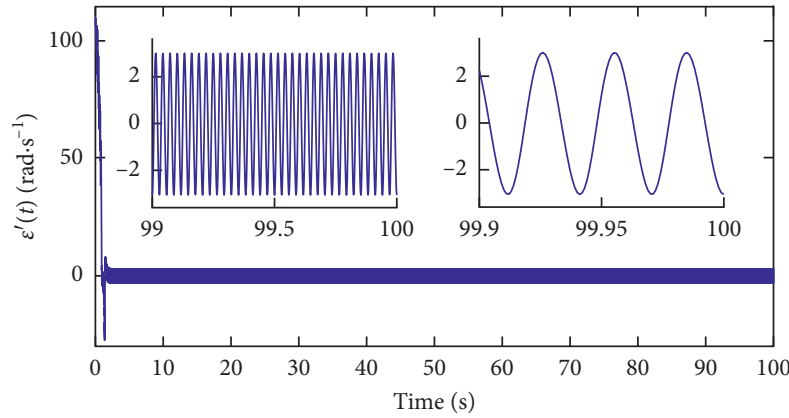

(a)

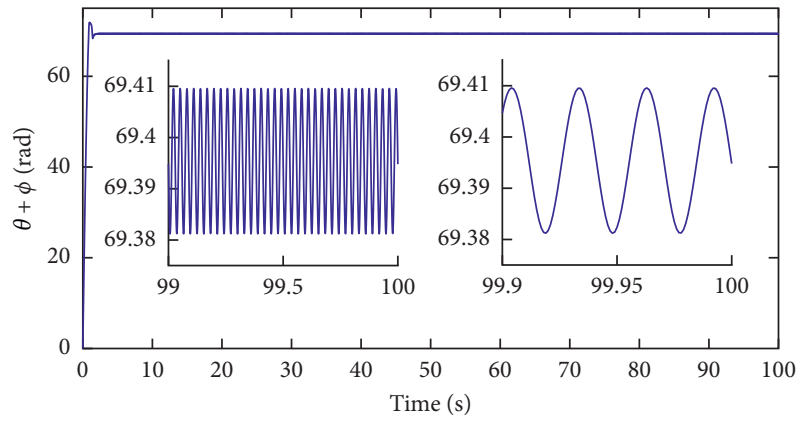

(b)

Figure 5: Simulation curve $\left(\omega=34 \pi, A_{x}=0.005\right.$, and $\left.A_{y}=0.001\right)$. (a) $\varepsilon^{\prime}(t)$ graph. (b) $\theta+\varphi(t)$ graph.

$$
\left|\frac{2\left(T_{e_{0}}-T_{f_{0}}-T_{d_{0}}\right)}{m r \omega^{2}\left(A_{x}-A_{y}\right)}\right| \approx 0.390<1 .
$$

The above values satisfy the criterion of synchronization, and the phase sum stable operating point is in the second quadrant. The simulation results are shown in Figure 6.

The exciter is in an electric working state, and the synchronization phase sum $\Delta a \approx 90.58 \mathrm{rad}$ (approximately $149^{\circ}$ in the range of $360^{\circ}$ ) is in the second quadrant.

Simulation 4. The parameters of the system are as follows: $\omega=34 \pi \mathrm{rad} / \mathrm{s}, A_{x}=0.001$, and $A_{y}=0.005$.

Verify the synchronization criterion according to the following parameters, when $\omega=34 \pi \mathrm{rad} / \mathrm{s}$, we get slip rate $s=-0.02, T_{e_{0}}=2.060, T_{f_{0}}=-f \omega \approx-0.214, T_{d_{0}}=-m r \omega^{2}$ $f d / 2 \approx-0.228$, and $m r \omega^{2}\left(A_{x}-A_{y}\right)=-9.127$, and we get

$$
\left|\frac{2\left(T_{e_{0}}-T_{f_{0}}-T_{d_{0}}\right)}{m r \omega^{2}\left(A_{x}-A_{y}\right)}\right| \approx 0.548<1 .
$$

The above values satisfy the criterion of synchronization, and the phase sum stable operating point is in the third quadrant. The simulation results are shown in Figure 7.

The exciter is in a feedback state, and the synchronization phase sum $\Delta \alpha \approx 97.67 \mathrm{rad}$ (approximately $196^{\circ}$ in the range of $360^{\circ}$ ) is in the third quadrant.

Simulation 5. The parameters of the system are as follows: $\omega=32.67 \pi \mathrm{rad} / \mathrm{s}, A_{x}=0.002$, and $A_{y}=0.001$. 


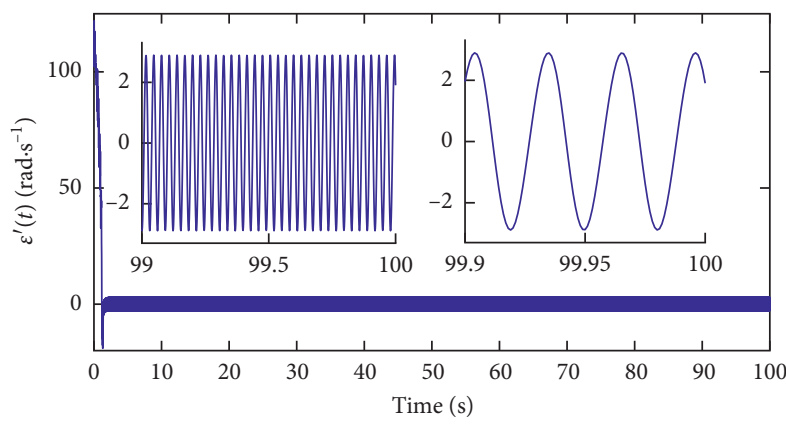

(a)

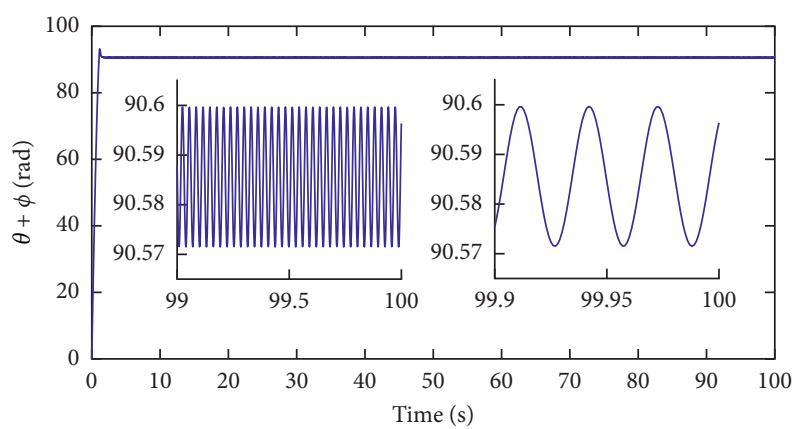

(b)

Figure 6: Simulation curve $\left(\omega=32.67 \pi, A_{x}=0.001\right.$, and $\left.A_{y}=0.005\right)$. (a) $\varepsilon^{\prime}(t)$ graph. (b) $\theta+\varphi(t)$ graph.

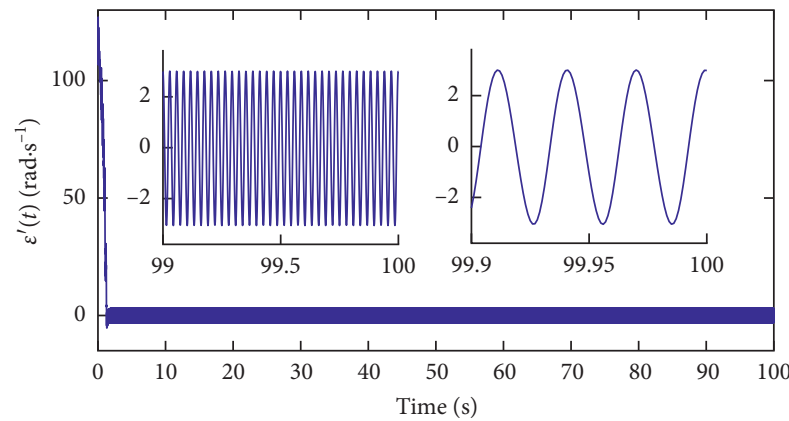

(a)

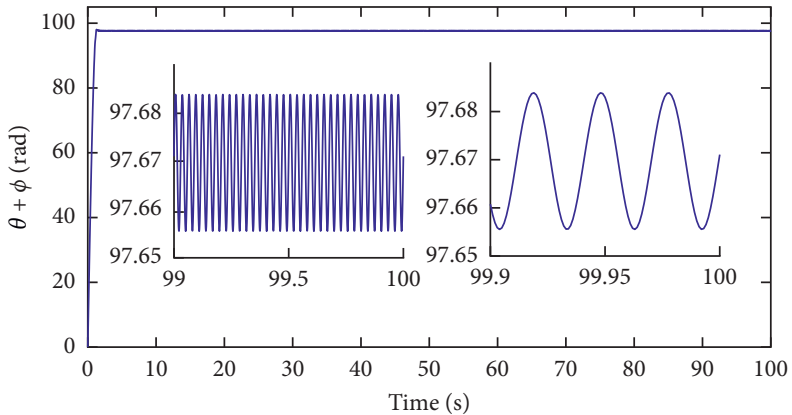

(b)

Figure 7: Simulation curve $\left(\omega=34 \pi, A_{x}=0.001\right.$, and $\left.A_{y}=0.005\right)$. (a) $\varepsilon^{\prime}(t)$ graph. (b) $\theta+\varphi(t)$ graph.

Verify the synchronization criterion according to the following parameters, when $\omega=32.67 \pi \mathrm{rad} / \mathrm{s}$, we get slip rate $s=0.02, T_{e_{0}}=-2.060, T_{f_{0}}=-f \omega \approx-0.205, T_{d_{0}}=-m r$ $\omega^{2} f d / 2 \approx-0.211$, and $\operatorname{mr} \omega^{2}\left(A_{x}-A_{y}\right)=2.107$, and we get

$$
\left|\frac{2\left(T_{e_{0}}-T_{f_{0}}-T_{d_{0}}\right)}{m r \omega^{2}\left(A_{x}-A_{y}\right)}\right| \approx 1.561>1
$$

The above values do not satisfy the criterion of synchronization. The simulation results are shown in Figure 8.

Simulation 6. The parameters of the system are as follows: $\omega=34 \pi \mathrm{rad} / s, A_{x}=0.001$, and $A_{y}=0.002$.

Verify the synchronization criterion according to the following parameters, when $\omega=34 \pi \mathrm{rad} / s$, we get slip rate $s=-0.02, \quad T_{e_{0}}=2.060, \quad T_{f_{0}}=-f \omega \approx-0.214, \quad T_{d_{0}}=$ $-m r \omega^{2} f d / 2 \approx-0.228$, and $m r \omega^{2}\left(A_{x}-A_{y}\right)=-2.282$, and we get

$$
\left|\frac{2\left(T_{e_{0}}-T_{f_{0}}-T_{d_{0}}\right)}{m r \omega^{2}\left(A_{x}-A_{y}\right)}\right| \approx 2.193>1 .
$$

The above values do not satisfy the criterion of synchronization. The simulation results are shown in Figure 9.

Remark 1. By combining the synchronization criterion and the stability criterion, the accurate quadrant range of the synchronization phase sum $\Delta \alpha$ can be determined.
When the exciter is operating in an electric working state,

$$
\left\{\begin{array}{c}
\omega<\omega_{0} \\
A_{x}>A_{y} .
\end{array}\right.
$$

The synchronous operating point phase sum $\Delta \alpha$ is in the fourth quadrant:

$$
\left\{\begin{array}{c}
\omega<\omega_{0}, \\
A_{x}<A_{y} .
\end{array}\right.
$$

The synchronous operating point phase sum $\Delta \alpha$ is in the second quadrant.

The conclusions can be as shown in Figure 10.

When the exciter is operating in a feedback state,

$$
\left\{\begin{array}{c}
\omega>\omega_{0}, \\
A_{x}>A_{y} .
\end{array}\right.
$$

The synchronous operating point phase sum $\Delta \alpha$ is in the first quadrant:

$$
\left\{\begin{array}{c}
\omega>\omega_{0}, \\
A_{x}<A_{y} .
\end{array}\right.
$$

The synchronous operating point phase sum $\Delta \alpha$ is in the third quadrant.

The conclusions can be as shown in Figure 11. 


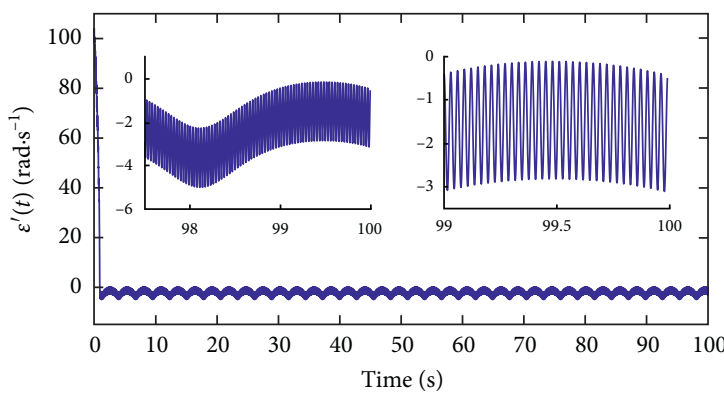

(a)

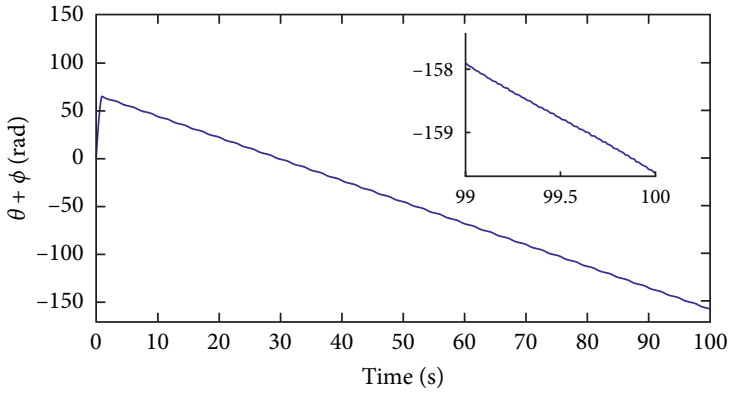

(b)

FIgURE 8: Simulation curve $\left(\omega=32.67 \pi, A_{x}=0.002\right.$, and $\left.A_{y}=0.001\right)$. (a) $\varepsilon^{\prime}(t)$ graph. (b) $\theta+\varphi(t)$ graph.

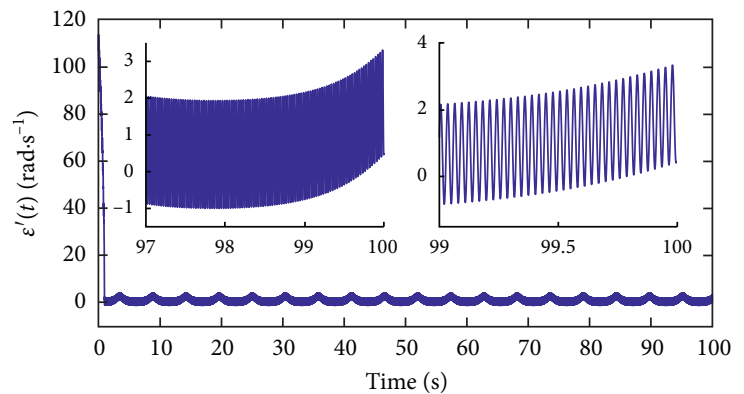

(a)

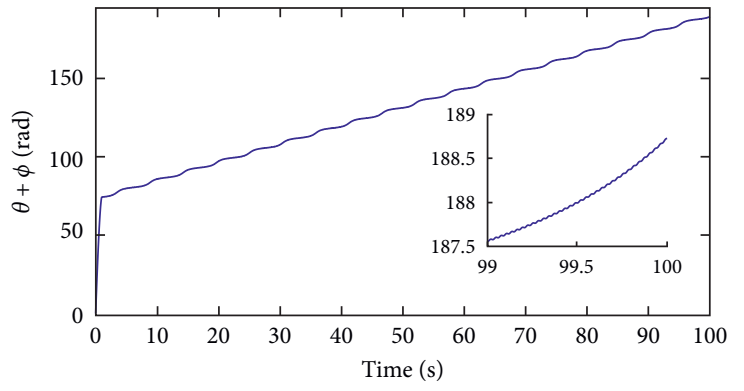

(b)

Figure 9: Simulation curve $\left(\omega=34 \pi, A_{x}=0.001\right.$, and $\left.A_{y}=0.002\right)$. (a) $\varepsilon^{\prime}(t)$ graph. (b) $\theta+\varphi(t)$ graph.

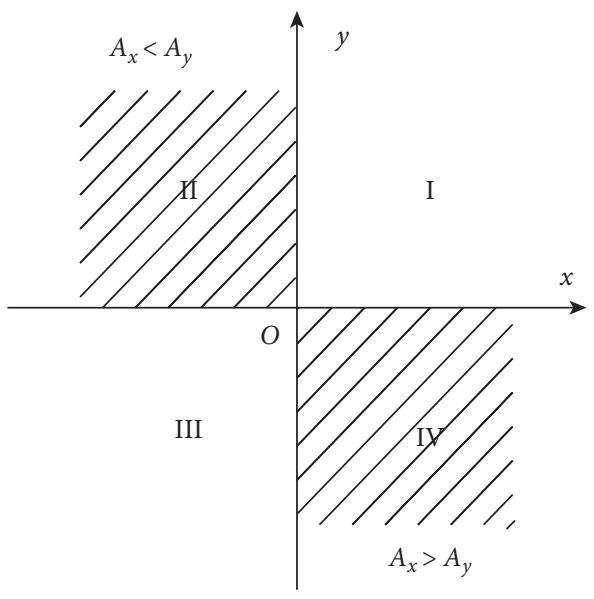

FIgURE 10: In the electric working state, the distribution of the synchronization phase sum $\Delta \alpha$.

Both the synchronization criterion and the stability criterion indicate that in order for the angular frequency of the counter-rotating eccentric rotor to be captured by the frequency of the composite vibrating field, it must first be satisfied that the amplitude $A_{x}$ and $A_{y}$ of the compound vibrating field in the $x$-direction and $y$-direction are not equal, that is, $A_{x} \neq A_{y}$.

This section verifies the criterion obtained by the above theory through 6 simulations. The setting of the simulation parameters is based on the relationship between the exciter working in the electric state and the feedback state, and the

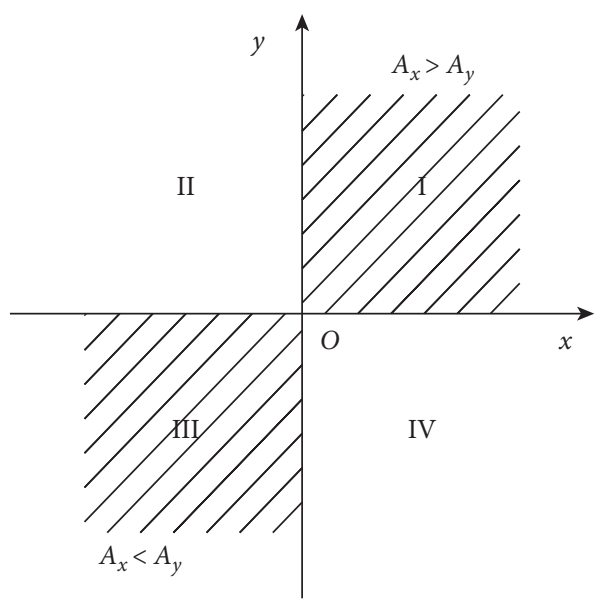

FIGURE 11: In the feedback state, the distribution of the synchronization phase sum $\Delta \alpha$.

amplitude in the $x$-direction and in the $y$-direction. The simulation results fully prove the effectiveness and feasibility of the two criteria. Compared with the simulation of the single corotating exciter vibrating model, the mean speed sum $\bar{\varepsilon}^{\prime}$ of the simulation of this model fluctuates greatly. The same conclusion can be obtained in the simulation of the same and opposite direction model of dual exciters. The setting of parameters in the criterion has an impact on the stability of the system, but how the relationship between specific parameters affects the system remains to be studied. 


\section{Conclusions}

In this paper, the concept of the compound vibrating field is proposed, which provides a new way for the study of complex vibration environment, and a vibrating system with a single counter-rotating exciter in a compound vibrating field is established.

The synchronization and stability analysis of the mathematical model of the vibrating system is carried out by integral mean method with small parameters and periodic coefficient, and finally, the synchronization criterion and the stability criterion of the vibratory synchronization of the counter-rotating exciter in the compound vibration field are obtained. The synchronization criterion and the stability criterion are used to judge the accurate quadrant range of the phase sum $\Delta \alpha$ when the system is synchronized and stable, and the criterion shows that the counter-rotating exciter cannot be synchronized in the compound vibration field with equal amplitude in the $x$ and $y$ directions. This has certain guiding significance for the parameter design of vibration testing equipment in a complex vibration environment.

\section{Data Availability}

The results are based on the theory calculation, and it is also verified by simulation with MATLAB/Simulink. The authors can provide the simulation model upon request.

\section{Conflicts of Interest}

The authors declare that there are no conflicts of interest regarding the publication of this paper.

\section{Acknowledgments}

The research was financially supported by the Special Fund of Chinese Central Government Guidance for Local Science and Technology Development (grant no. [2016] 4006). The authors thank CINTEC Heavy Equipment (China) Co. Ltd. for its support.

\section{References}

[1] B. Wen, "Recent development of vibration utilization engineering," Frontiers of Mechanical Engineering in China, vol. 3, pp. 1-9, 2008.

[2] C. Huygens, Horologium Oscilatorium, Apud F. Muguet, Paris, France, 1673.

[3] B. van der Pol, "Theory of the amplitude of free and forced triode vibrations," Radio Rev.vol. 1, pp. 701-710, 1920.

[4] L. Rayleigh and N. H. Nachtrieb, "The theory of sound," Physics Today, vol. 10, no. 1, pp. 32-34, 1957.

[5] I. I. Blekhman, "The problem of synchronization of dynamical systems," Journal of Applied Mathematics and Mechanics, vol. 28, no. 2, pp. 239-265, 1964.

[6] I. I. Blekhman, Synchronization in Science and Technology, ASME Press, New York, NW, USA, 1988.

[7] B. Wen and L. Guan, "The synchronous theory of a selfsynchronous vibrating machine with two masses in plane motion," Journal of Northeastern University, vol. 02, pp. 1526, 1979.

[8] B. Wen, "Some new results to research on "vibration synchronization theory' with its application," Journal of Vibration and Shock, no. 3, pp. 1-10, 1983.

[9] B. Wen and X. Lin, "Vibratory synchronization transmission and its industry applications," Journal of Mechanical Engineering, vol. 20, no. 3, pp. 26-42, 1984.

[10] C. Zhao, H. Zhu, R. Wang, and B. Wen, "Synchronization of two non-identical coupled exciters in a non-resonant vibrating system of linear motion. Part I: theoretical analysis," Shock and Vibration, vol. 16, no. 5, Article ID 259149, 2008.

[11] C. Zhao, K. Liu, X. Ye et al., "Self-synchronization theory of a vibrating system with two-motor drives rotating in opposite directions," Journal of Mechanical Engineering, vol. 45, no. 09, pp. 24-30, 2009.

[12] C. Zhao, B. He, J. Liu, Y. Han, and B. Wen, "Design method of dynamic parameters of a self-synchronization vibrating system with dual mass," Proceedings of the Institution of Mechanical Engineers - Part K: Journal of Multi-Body Dynamics, vol. 232, no. 1, pp. 3-20, 2018.

[13] I. I. Blekhman, A. L. Fradkov, H. Nijmeijer, and A. Y. Pogromsky, "On self-synchronization and controlled synchronization," Systems \& Control Letters, vol. 31, no. 5, pp. 299-305, 1997.

[14] J. M. Balthazar, "Short comments on self-synchronization of two non-ideal sources supported by a flexible portal frame structure," Journal of Vibration and Control, vol. 10, no. 12, pp. 1739-1748, 2004.

[15] J. M. Balthazar, J. L. P. Felix, and R. M. Brasil, "Some comments on the numerical simulation of self-synchronization of four non-ideal exciters," Applied Mathematics and Computation, vol. 164, no. 2, pp. 615-625, 2004.

[16] J. L. P. Felix, J. M. Balthazar, and M. J. H. Dantas, "On energy pumping, synchronization and beat phenomenon in a nonideal structure coupled to an essentially nonlinear oscillator," Nonlinear Dynamics, vol. 56, no. 1-2, pp. 1-11, 2009.

[17] X. Zhang, B. Wen, and C. Zhao, "Vibratory synchronization transmission of two exciters in a super-resonant vibrating system," Journal of Mechanical Science and Technology, vol. 28, no. 6, pp. 2049-2058, 2014.

[18] X. Zhang, B. Wen, and C. Zhao, "Vibratory synchronization transmission of a cylindrical roller in a vibrating mechanical system excited by two exciters," Mechanical Systems and Signal Processing, vol. 96, pp. 88-103, 2017.

[19] X. Zhang, C. Li, Z. Wang, and S. Cui, "Synchronous stability of four homodromy vibrators in a vibrating system with double resonant types," Shock and Vibration, vol. 2018, Article ID 9641231, 20 pages, 2018.

[20] X. Kong, C. Chen, and B. Wen, "Composite synchronization of three eccentric rotors driven by induction motors in a vibrating system," Mechanical Systems and Signal Processing, vol. 102, pp. 158-179, 2018.

[21] X. Kong and B. Wen, "Composite synchronization of a four eccentric rotors driven vibration system with a mass-spring rigid base," Journal of Sound and Vibration, vol. 427, pp. 63-81, 2018.

[22] D. Gu, X. Zhang, J. Zhang, Y. Liu, and B. We, "Synchronization and coupling dynamic characteristics of an exciter and two cylindrical rollers in a vibrating system," Journal of Sound and Vibration, vol. 456, pp. 353-373, 2019.

[23] Y. Liu, X. Zhang, D. Gu, L. Jia, and B. Wen, "Synchronization of a dual-mass vibrating system with two exciters," Shock and Vibration, vol. 2020, Article ID 9345652, 12 pages, 2020. 
[24] X. Liao, Mathematical Theory and Application of Stability, Center-China Normal University Press, Wuhan, China, 2001.

[25] X. Qian, B. Dai, and K. Liu, Nonlinear Differential Equations: Theory, Method and Application, Hunan University Press, Changsha, China, 2006.

[26] M. Su, R. Li, Z. Xie, and Z. Jimming, "Theory of frequency captured of eccentric rotor by vibration environment with same direction," Shock and Vibration, vol. 2021, Article ID 8892919, 8 pages, 2021.

[27] Y. Han, B. He, and C. Zhao, "Research on theory of dynamic coupling for self-synchronization of dual-mass vibrating screen," Journal of Yanshan University, vol. 41, no. 05, pp. 413-418+427, 2017.

[28] C. Xiaoxin, "Characteristic exponents for second order periodic linear differential equation," Annals of Differential Equations, no. 2, pp. 107-118, 1997.

[29] J. Shi, "On stability of two order linear differential equations with periodic coefficient," Acta Mathematica Scientia, vol. 01, pp. 130-139, 2000. 